\title{
Approximate Reduction of Dynamical Systems
}

\author{
Paulo Tabuada, Aaron D. Ames, Agung Julius and George Pappas
}

\begin{abstract}
The reduction of dynamical systems has a rich history, with many important applications related to stability, control and verification. Reduction is typically performed in an "exact" manner-as is the case with mechanical systems with symmetry-which, unfortunately, limits the type of systems to which it can be applied. The goal of this paper is to consider a more general form of reduction, termed approximate reduction, in order to extend the class of systems that can be reduced. Using notions related to incremental stability, we give conditions on when a dynamical system can be projected to a lower dimensional space while providing hard bounds on the induced errors, i.e., when it is behaviorally similar to a dynamical system on a lower dimensional space. These concepts are illustrated on a series of examples.
\end{abstract}

\section{INTRODUCTION}

Modeling is an essential part of many engineering disciplines and often a key ingredient for successful designs. Although it is widely recognized that models are only approximate descriptions of reality, their value lies precisely on the ability to describe, within certain bounds, the modeled phenomena. In this paper we consider modeling of closed loop control systems, i.e., differential equations, with the purpose of simplifying the analysis of these systems. The goal of this paper is to reduce the dimension of the differential equations being analyzed while providing hard bounds on the introduced errors. One promising application of these techniques is to the verification of hybrid systems, which is currently constrained by the complexity of high dimensional differential equations.

Reducing differential equations-and in particular mechanical systems - is a subject with a long and rich history. The first form of reduction was discovered by Routh in the 1890's; over the years, geometric reduction has become an academic field in it self. One begins with a differential equation with certain symmetries, i.e., it is invariant under the action of a Lie group on the phase space. Using these symmetries, one can reduce the dimensionality of the phase space (by "dividing" out by the symmetry group) and define a corresponding differential equation on this reduced phase

This research was partially supported by the National Science Foundation, EHS award 0509313 and CCR award 0225610.

P. Tabuada is with the Department of Electrical Engineering, University of California at Los Angeles, Los Angeles, CA 90095. E-mail: tabuada@ee.ucla.edu Web: http: / / www. ee. ucla. edu/ tabuada

A. D. Ames is with the Control and Dynamical Systems Department, California Institute of Technology, Pasadena, CA 91125. E-mail: amesacds.caltech.edu Web: http: / / www. cds. caltech. edu/ ames

Agung Julius and George Pappas are with the Department of Electrical and Systems Engineering, University of Pennsylvania, Philadelphia, PA 19104. E-mail: \{ agungaseas.upenn.edu, pappasg@ee.upenn.edu\} space. The main result of geometric reduction is that one can understand the behavior of the full-order system in terms of the behavior of the reduced system and vice versa [MW74], [vdS81], [BKMM96]. While this form of "exact" reduction is very elegant, the class of systems for which this procedure can be applied is actually quite small. This indicates the need for a form of reduction that is applicable to a wider class of systems and, while not being exact, is "close enough."

In systems theory, reduced order modeling has also been extensively studied under the name of model reduction [BDG96], [ASG00]. Contrary to model reduction where approximation is measured using $L^{2}$ norms we are interested in $L^{\infty}$ norms. The guarantees provided by $L^{\infty}$ norms are more natural when applications to safety verification are of interest.

We develop our results in the framework of incremental stability and our main result is in the spirit of existing stability results for cascade systems that proliferate the Inputto-State Stability (ISS) literature.

\section{PRELIMINARIES}

A continuous function $\gamma: \mathbb{R}_{0}^{+} \rightarrow \mathbb{R}_{0}^{+}$, is said to belong to class $\mathcal{K}_{\infty}$ if it is strictly increasing, $\gamma(0)=0$ and $\gamma(r) \rightarrow \infty$ as $r \rightarrow \infty$. A continuous function $\beta: \mathbb{R}_{0}^{+} \times \mathbb{R}_{0}^{+} \rightarrow \mathbb{R}_{0}^{+}$is said to belong to class $\mathcal{K} \mathcal{L}$ if, for each fixed $s$, the map $\beta(r, s)$ belongs to class $\mathcal{K}_{\infty}$ with respect to $r$ and, for each fixed $r$, the map $\beta(r, s)$ is decreasing with respect to $s$ and $\beta(r, s) \rightarrow 0$ as $s \rightarrow \infty$.

For a smooth function $\varphi: \mathbb{R}^{n} \rightarrow \mathbb{R}^{m}$ we denote by $T \varphi$ the tangent map to $\varphi$ and by $T_{x} \varphi$ the tangent map to $\varphi$ at $x \in \mathbb{R}^{n}$. We will say that $\varphi$ is a submersion at $x \in \mathbb{R}^{n}$ if $T_{x} \varphi$ is surjective and that $T \varphi$ is a submersion if it is a submersion at every $x \in \mathbb{R}^{n}$. When $\varphi$ is a submersion we will also use the notation $\operatorname{ker}(T \varphi)$ to denote the distribution:

$$
\operatorname{ker}(T \varphi)=\left\{X: \mathbb{R}^{n} \rightarrow \mathbb{R}^{n} \mid T_{x} \varphi \cdot X(x)=0\right\} .
$$

Given a point $x \in \mathbb{R}^{n},|x|$ will denote the usual Euclidean norm while $\|f\|$ will denote $\operatorname{ess} \sup _{t \in[0, \tau]}|f(t)|$ for any given function $f:[0, \tau] \rightarrow \mathbb{R}^{n}, \tau>0$.

\section{A. Dynamical and control systems}

In this paper we shall restrict our attention to dynamical and control systems defined on Euclidean spaces.

Definition 1: A vector field is a pair $\left(\mathbb{R}^{n}, X\right)$ where $X$ is a smooth map $X: \mathbb{R}^{n} \rightarrow \mathbb{R}^{n}$. A smooth curve $\mathbf{x}(\cdot, x)$ : $I \rightarrow \mathbb{R}^{n}$, defined on an open subset $I$ of $\mathbb{R}$ including the origin, is said to be a trajectory of $\left(\mathbb{R}^{n}, X\right)$ if the following two conditions hold:

1) $\mathbf{x}(0, x)=x$; 
2) $\frac{d}{d t} \mathbf{x}(t, x)=X(\mathbf{x}(t, x))$ for all $t \in I$.

A control system can be seen as an under-determined vector field.

Definition 2: A control system is a triple $\left(\mathbb{R}^{n}, \mathbb{R}^{m}, F\right)$ where $F$ is a smooth map $F: \mathbb{R}^{n} \times \mathbb{R}^{m} \rightarrow \mathbb{R}^{n}$. A smooth curve $\mathbf{x}_{\mathbf{u}}(\cdot, x): I \rightarrow \mathbb{R}^{n}$, defined on an open subset $I$ of $\mathbb{R}$ including the origin, is said to be a trajectory of $\left(\mathbb{R}^{n}, \mathbb{R}^{m}, F\right)$ if there exists a smooth curve $\mathbf{u}: I \rightarrow \mathbb{R}^{m}$ such that the following two conditions hold:

1) $\mathbf{x}_{\mathbf{u}}(0, x)=x$

2) $\frac{d}{d t} \mathbf{x}_{\mathbf{u}}(t, x)=F\left(\mathbf{x}_{\mathbf{u}}(t, x), \mathbf{u}(t)\right)$ for almost all $t \in I$.

\section{EXACT REDUCTION}

For some dynamical systems described by a vector field $X$ on $\mathbb{R}^{n}$ it is possible to replace $X$ by a vector field $Y$ describing the dynamics of the system on a lower dimensional space, $\mathbb{R}^{m}$, while retaining much of the information in $X$. When this is the case we say that $X$ can be reduced to $Y$. This idea of (exact) reduction is captured by the notion of $\varphi$-related vector fields.

Definition 3: Let $\varphi: \mathbb{R}^{n} \rightarrow \mathbb{R}^{m}$ be a smooth map. The vector field $\left(\mathbb{R}^{n}, X\right)$ is said to be $\varphi$-related to the vector field $\left(\mathbb{R}^{m}, Y\right)$ if:

$$
T \varphi \cdot X=Y \circ \varphi
$$

The following proposition, proved in [AMR88], characterizes $\varphi$-related vector fields in terms of their trajectories.

Proposition 1: The vector field $\left(\mathbb{R}^{n}, X\right)$ is $\varphi$-related to the vector field $\left(\mathbb{R}^{m}, Y\right)$, for some smooth map $\varphi: \mathbb{R}^{n} \rightarrow \mathbb{R}^{m}$, iff:

$$
\varphi \circ \mathbf{x}(t, x)=\mathbf{y}(t, \varphi(x)),
$$

where $\mathbf{x}(t, x)$ and $\mathbf{y}(t, y)$ are the trajectories of vector fields $X$ and $Y$, respectively.

For $\varphi$-related vector fields, we can replace the study of trajectories $\mathbf{x}(\cdot, x)$ with the study of trajectories $\mathbf{y}(\cdot, \varphi(x))$ living on the lower dimension space $\mathbb{R}^{m}$. In particular, formal verification of $X$ can be performed on $Y$ whenever the relevant sets describing the verification problem can also be reduced to $\mathbb{R}^{m}$.

If a vector field and a submersion $\varphi$ are given we can use the following result, proved in [MSVS85], to determine the existence of $\varphi$-related vector fields.

Proposition 2: Let $\left(\mathbb{R}^{n}, X\right)$ be a vector field and $\varphi$ : $\mathbb{R}^{n} \rightarrow \mathbb{R}^{m}$ a smooth submersion. There exists a vector field $\left(\mathbb{R}^{m}, Y\right)$ that is $\varphi$-related to $\left(\mathbb{R}^{n}, X\right)$ iff:

$$
[\operatorname{ker}(T \varphi), X] \subseteq \operatorname{ker}(T \varphi)
$$

In an attempt to enlarge the class of vector fields that can be reduced we introduce, in the next section, an approximate notion of reduction.

\section{Approximate Reduction}

The generalization of Definition 3 proposed in this section requires a decomposition of $\mathbb{R}^{n}$ of the form $\mathbb{R}^{n}=\mathbb{R}^{m} \times \mathbb{R}^{k}$. Associated with this decomposition are the canonical projections $\pi_{m}: \mathbb{R}^{n} \rightarrow \mathbb{R}^{m}$ and $\pi_{k}: \mathbb{R}^{n} \rightarrow \mathbb{R}^{k}$ taking $\mathbb{R}^{n} \ni x=(y, z) \in \mathbb{R}^{m} \times \mathbb{R}^{k}$ to $\pi_{m}(x)=y$ and $\pi_{k}(x)=z$, respectively.
Definition 4: The vector field $\left(\mathbb{R}^{n}, X\right)$ is said to be approximately $\pi_{m}$-related to the vector field $\left(\mathbb{R}^{m}, Y\right)$ if there exists a class $\mathcal{K}_{\infty}$ function $\gamma$ such that the following estimate holds:

$$
\left|\pi_{m} \circ \mathbf{x}(t, x)-\mathbf{y}\left(t, \pi_{m}(x)\right)\right| \leq \gamma\left(\left|\pi_{k}(x)\right|\right) .
$$

Note that when $X$ and $Y$ are $\pi_{m}$-related we have:

$$
\left|\pi_{m} \circ \mathbf{x}(t, x)-\mathbf{y}\left(t, \pi_{m}(x)\right)\right|=0,
$$

which implies (3). Definition 4 can thus be seen as a generalization of exact reduction captured by Definition 3 .

Although the bound on the gap between the projection of the original trajectory $\mathbf{x}$ and the trajectory $\mathbf{y}$ of the approximate reduced system is a function of $x$, in concrete applications the initial conditions are typically restricted to a bounded set of interest. The following result has interesting implications in these situations.

Proposition 3: If $\left(\mathbb{R}^{n}, X\right)$ is approximately $\pi_{m}$-related to $\left(\mathbb{R}^{m}, Y\right)$ then for any compact set $C \subseteq \mathbb{R}^{n}$ there exists a $\delta>0$ such that for all $x \in C$ the following estimate holds:

$$
\left|\pi_{m} \circ \mathbf{x}(t, x)-\mathbf{y}\left(t, \pi_{m}(x)\right)\right| \leq \delta .
$$

Proof: Let $\delta=\max _{x \in C} \gamma\left(\left|\pi_{k}(x)\right|\right)$. The scalar $\delta$ is well defined since $\gamma\left(\left|\pi_{k}(\cdot)\right|\right)$ is a continuous map and $C$ is compact.

From a practical point of view, approximate reduction is only a useful concept if it admits characterizations that are simple to check. In order to derive such characterizations we need to review several notions of incremental stability.

\section{A. Incremental stability}

In this subsection we review two notions of incremental stability which will be fundamental in proving the main contribution of this paper. We follow [BM00] and [Ang02].

Definition 5: A control system $\left(\mathbb{R}^{n}, \mathbb{R}^{m}, F\right)$ is said to be incrementally uniformly bounded-input-bounded-state stable (IUBIBSS) if there exist two class $\mathcal{K}_{\infty}$ functions $\gamma_{1}$ and $\gamma_{2}$ such that for each $x_{1}, x_{2} \in \mathbb{R}^{n}$ and for each pair of smooth curves $\mathbf{u}_{1}, \mathbf{u}_{2}: I \rightarrow \mathbb{R}^{m}$ the following estimate holds:

$\left|\mathbf{x}_{\mathbf{u}_{1}}\left(t, x_{1}\right)-\mathbf{x}_{\mathbf{u}_{\mathbf{2}}}\left(t, x_{2}\right)\right| \leq \gamma_{1}\left(\left|x_{1}-x_{2}\right|\right)+\gamma_{2}\left(\left\|\mathbf{u}_{1}-\mathbf{u}_{2}\right\|\right)$

for all $t \in I$.

In general it is difficult to establish IUBIBSS directly. A sufficient condition is given by the existence of an IUBIBSS Lyapunov function. Note however that IUBIBSS only implies the existence of a IUBIBSS Lyapunov function with very weak regularity conditions [BM00].

Definition 6: A $C^{1}$ function $V: \mathbb{R}^{n} \times \mathbb{R}^{n} \rightarrow \mathbb{R}_{0}^{+}$is said to be an IUBIBSS Lyapunov function for control system $\left(\mathbb{R}^{n}, \mathbb{R}^{m}, F\right)$ if there exist a $\xi>0$ and class $\mathcal{K}_{\infty}$ functions $\underline{\alpha}, \bar{\alpha}$, and $\mu$ such that:

1) For $\left|x_{1}-x_{2}\right| \geq \xi$,

$$
\underline{\alpha}\left(\left|x_{1}-x_{2}\right|\right) \leq V\left(x_{1}, x_{2}\right) \leq \bar{\alpha}\left(\left|x_{1}-x_{2}\right|\right) ;
$$

2) $\mu(r) \geq r+\xi$ for $r \in \mathbb{R}_{0}^{+}$;

3) $\left|x_{1}-x_{2}\right| \geq \mu\left(\left|u_{1}-u_{2}\right|\right) \quad \Longrightarrow \quad \dot{V} \leq 0$. 
A stronger notion than IUBIBSS is incremental input-tostate stability.

Definition 7: A control system $\left(\mathbb{R}^{n}, \mathbb{R}^{m}, F\right)$ is said to be incrementally input-to-state stable (IISS) if there exist a class $\mathcal{K} \mathcal{L}$ function $\beta$ and a class $\mathcal{K}_{\infty}$ function $\gamma$ such that for each $x_{1}, x_{2} \in \mathbb{R}^{n}$ and for each pair of smooth curves $\mathbf{u}_{1}, \mathbf{u}_{2}: I \rightarrow \mathbb{R}^{m}$ the following estimate holds:

$\left|\mathbf{x}_{\mathbf{u}_{\mathbf{1}}}\left(t, x_{1}\right)-\mathbf{x}_{\mathbf{u}_{\mathbf{2}}}\left(t, x_{2}\right)\right| \leq \beta\left(\left|x_{1}-x_{2}\right|, t\right)+\gamma\left(\left\|\mathbf{u}_{1}-\mathbf{u}_{2}\right\|\right)$

Since $\beta$ is a decreasing function of $t$ we immediately see that (6) implies (5) with $\gamma_{1}(r)=\beta(r, 0)$ and $\gamma_{2}(r)=\gamma(r)$, $r \in \mathbb{R}_{0}^{+}$. Once again, IISS is implied by the existence of an IISS Lyapunov function. See [Ang02] for a converse result when the inputs take values in a compact set.

Definition 8: A $C^{1}$ function $V: \mathbb{R}^{n} \times \mathbb{R}^{n} \rightarrow \mathbb{R}_{0}^{+}$is said to be an IISS Lyapunov function for the control system $\left(\mathbb{R}^{n}, \mathbb{R}^{m}, F\right)$ if there exist class $\mathcal{K}_{\infty}$ functions $\underline{\alpha}, \bar{\alpha}, \alpha$, and $\mu$ such that:

1) $\underline{\alpha}\left(\left|x_{1}-x_{2}\right|\right) \leq V\left(x_{1}, x_{2}\right) \leq \bar{\alpha}\left(\left|x_{1}-x_{2}\right|\right)$;

2) $\left|x_{1}-x_{2}\right| \geq \mu\left(\left|u_{1}-u_{2}\right|\right) \Longrightarrow \dot{V} \leq-\alpha\left(\left|x_{1}-x_{2}\right|\right)$.

\section{B. Fiberwise stability}

In addition to incremental stability we will also need a notion of partial stability.

Definition 9: A vector field $\left(\mathbb{R}^{n}, X\right)$ is said to be fiberwise stable with respect to $\mathbb{R}^{k}$ if there exists a class $\mathcal{K}_{\infty}$ function $\gamma$ such that the following estimate holds:

$$
\left\|\pi_{k}(\mathbf{x}(\cdot, x))\right\| \leq \gamma\left(\left|\pi_{k}(x)\right|\right) .
$$

Fiberwise stability can be checked with the help of the following result.

Lemma 1: A vector field $\left(\mathbb{R}^{n}, X\right)$ is fiberwise stable with respect to $\mathbb{R}^{k}$ if there exist two $\mathcal{K}_{\infty}$ functions, $\underline{\alpha}$ and $\bar{\alpha}$, and a function $V: \mathbb{R}^{n} \rightarrow \mathbb{R}$ such that:

1) $\underline{\alpha}\left(\left|\pi_{k}(x)\right|\right) \leq V(x) \leq \bar{\alpha}\left(\left|\pi_{k}(x)\right|\right)$,

2) $\dot{\dot{V}} \leq 0$.

\section{Existence of approximate reductions}

In this subsection we prove the main result providing sufficient conditions for the existence of approximate reductions.

Theorem 1: Let $\left(\mathbb{R}^{n}, X\right)$ be a fiberwise stable vector field with respect to $\mathbb{R}^{k}$ and let $F=T \pi_{m} \cdot X: \mathbb{R}^{m} \times \mathbb{R}^{k} \rightarrow \mathbb{R}^{m}$, viewed as a control system with state space $\mathbb{R}^{m}$, be IUBIBSS. Then, the vector field $\left(\mathbb{R}^{m}, Y\right)$ defined by:

$$
Y(y)=T_{(y, z)} \pi_{m} \cdot X(y, 0)=F(y, 0)
$$

is approximately $\pi_{m}$-related to $\left(\mathbb{R}^{n}, X\right)$.

Proof: By assumption $Y(y)=T_{(y, z)} \pi_{m} \cdot X(y, 0)$ is IUBIBSS with respect to $\mathbb{R}^{k}$ so that we have:

$$
\left|\mathbf{y}_{\mathbf{v}_{1}}\left(t, y_{1}\right)-\mathbf{y}_{\mathbf{v}_{2}}\left(t, y_{2}\right)\right| \leq \gamma_{1}\left(\left|y_{1}-y_{2}\right|\right)+\gamma_{2}\left(\left\|\mathbf{v}_{1}-\mathbf{v}_{2}\right\|\right)
$$

In particular we can take

$$
y_{1}=y_{2}=\pi_{m}(x), \quad \mathbf{v}_{1}=\pi_{k} \circ \mathbf{x}(\cdot, x), \quad \mathbf{v}_{2}=0,
$$

to get:

$$
\begin{aligned}
\mid \pi_{m} \circ \mathbf{x} & (t, x)-\mathbf{y}\left(t, \pi_{m}(x)\right) \mid \\
& =\left|\mathbf{y}_{\pi_{k} \circ \mathbf{x}(t, x)}\left(t, \pi_{m}(x)\right)-\mathbf{y}_{0}\left(t, \pi_{m}(x)\right)\right| \\
& =\left|\mathbf{y}_{\mathbf{v}_{1}}\left(t, \pi_{m}(x)\right)-\mathbf{y}_{0}\left(t, \pi_{m}(x)\right)\right| \\
& \leq \gamma_{2}\left(\left\|\mathbf{v}_{1}\right\|\right)=\gamma_{2}\left(\left\|\pi_{k} \circ \mathbf{x}(\cdot, x)\right\|\right) .
\end{aligned}
$$

But it follows from fiber stability of $X$ with respect to $\mathbb{R}^{k}$ that

$$
\left\|\pi_{k} \circ \mathbf{x}(\cdot, x)\right\| \leq \gamma\left(\left|\pi_{k}(x)\right|\right) .
$$

We thus have:

$$
\left|\pi_{m} \circ \mathbf{x}(t, x)-\mathbf{y}\left(t, \pi_{m}(x)\right)\right| \leq \gamma_{2} \circ \gamma\left(\left|\pi_{k}(x)\right|\right),
$$

which concludes the proof since $\gamma_{2} \circ \gamma$ is a class $\mathcal{K}_{\infty}$ function.

Theorem 1 shows that sufficient conditions for approximate reduction can be given in terms of ISS-like Lyapunov functions and how reduced system can be constructed. Before illustrating Theorem 1 with several examples in the next section we present an important corollary.

Corollary 1: Let $\left(\mathbb{R}^{n}, X\right)$ and $\left(\mathbb{R}^{m}, Y\right)$ be vector fields satisfying the assumptions of Theorem 1. Then, for any compact set $C \subseteq \mathbb{R}^{n}$ there exists a $\delta>0$ such that for any $x \in C$ and $y \in \pi_{m}(C)$ the following estimate holds:

$$
\left|\pi_{m} \circ \mathbf{x}(t, x)-\mathbf{y}(t, y)\right| \leq \delta
$$

Proof: Using the same proof as for Theorem 1, except picking $y_{1}=\pi_{m}(x)$ and $y_{2}=y$, it follows that:

$$
\left|\pi_{m} \circ \mathbf{x}(t, x)-\mathbf{y}(t, y)\right| \leq \gamma_{1}\left(\left|\pi_{m}(x)-y\right|\right)+\gamma\left(\left|\pi_{k}(x)\right|\right) .
$$

The bound $\delta$ is now given by:

$$
\delta=\max _{(x, y) \in C \times \pi_{m}(C)}\left(\gamma_{1}\left(\left|\pi_{m}(x)-y\right|\right)+\gamma\left(\left|\pi_{k}(x)\right|\right)\right)
$$

which is well defined since $C \times \pi_{m}(C)$ is compact.

This result has important consequences for verification. Given a set of initial conditions $S \subset \mathbb{R}^{m}$, the set of points reachable under trajectories of $Y$ from initial conditions in $S$ can be over-approximated by enclosing a single trajectory of $Y$, starting at any point in $S$, by a tube of radius $\delta$.

\section{EXAMPLES}

In this section, we consider examples that illustrate the usefulness of approximate reduction.

Example 1: As a first example we consider the ball in a rotating hoop with friction, as described in Chapter 2 of [MR99]. For this example, there are the following parameters:

$$
\begin{aligned}
m & =\text { mass of the ball, } \\
R & =\text { Radius of the hoop } \\
g & =\text { acceleration due to gravity } \\
\mu & =\text { friction constant for the ball. }
\end{aligned}
$$

The equations of motion are given by:

$$
\begin{aligned}
\dot{\omega} & =-\frac{\mu}{m} \omega+\xi^{2} \sin \theta \cos \theta-\frac{g}{R} \sin \theta \\
\dot{\theta} & =\omega
\end{aligned}
$$



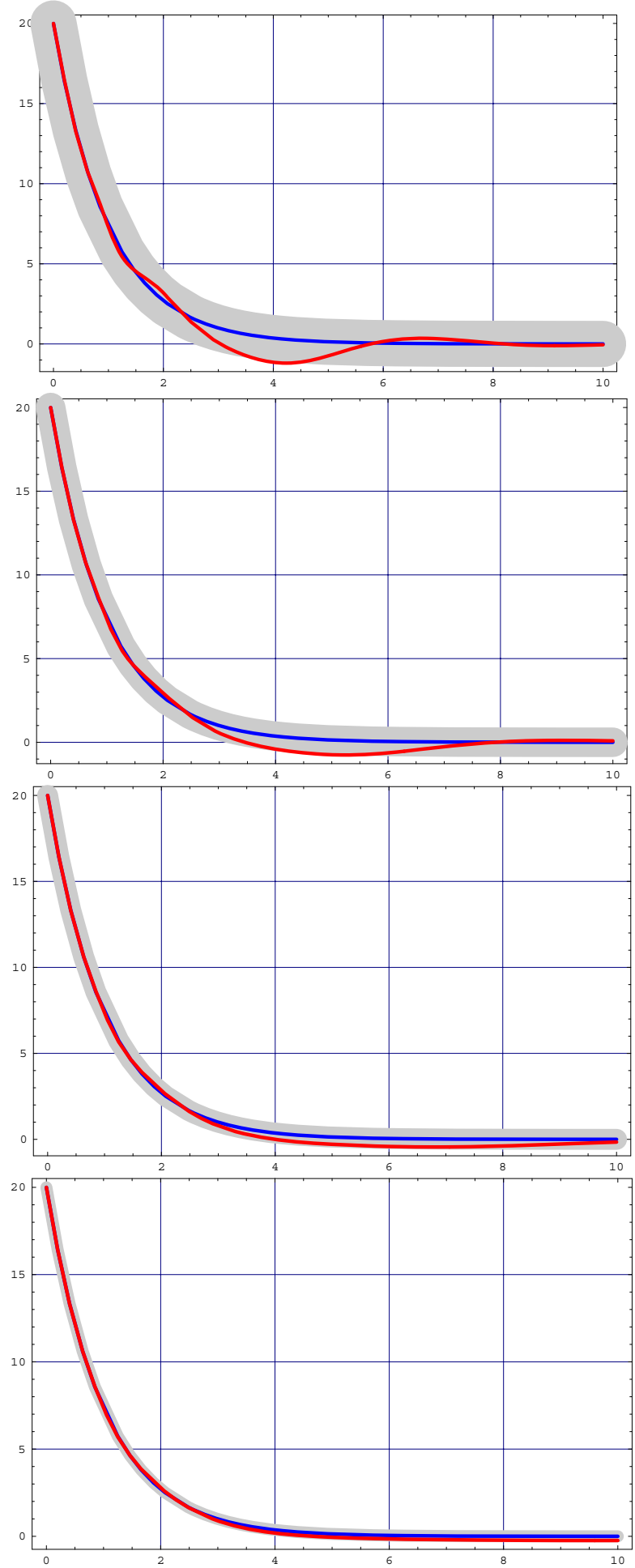

Fig. 1. A trajectory of the full order system (red) vs. a trajectory for the reduced system (blue) for $R=5,10,20,40$ (from top to bottom, respectively). where $\theta$ is the angular position of the ball and $\omega$ is its angular velocity.

If $\pi_{\omega}: \mathbb{R}^{2} \rightarrow \mathbb{R}$ is the projection $\pi_{\omega}(\omega, \theta)=\omega$, then according to Proposition 2 there exists no vector field $Y$ on $\mathbb{R}$ which is $\pi_{\omega}$-related to $X$ (as defined by (7)). However, we will show that $Y(\omega)=T_{(\omega, \theta)} \pi_{\omega} \cdot X(\omega, 0)$ is approximate $\pi_{\omega}$-related to $X$.

First, we use:

$$
V=\frac{1}{2} m R^{2} \omega^{2}+m g R(1-\cos \theta)-\frac{1}{2} m R^{2} \xi^{2} \sin ^{2} \theta
$$

as a Lyapunov function to show that (7) is stable. Note that $V(\omega, \theta)=0$ for $(\omega, \theta)=(0,0)$ and $V(\omega, \theta)>0$ for $(\omega, \theta) \neq$ $(0,0)$ provided that $R \xi^{2}<g$. Computing the time derivative of $V$ we obtain:

$$
\dot{V}=-\mu R^{2} \omega^{2} \leq 0,
$$

thus showing stability of (7). We now consider a compact set $C$ invariant under the dynamics and restrict our analysis to initial conditions in this set. Such a set can be constructed, for example, by taking $\left\{x \in \mathbb{R}^{2} \mid V(x) \leq c\right\}$ for some positive constant $c$. Note that stability of (7) implies fiberwise stability on $C$ since $\pi_{m}(C)$ is compact.

To apply Theorem 1 we only need to show that:

$$
T_{(\omega, \theta)} \pi_{\omega} \cdot X(\omega, \theta)=-\frac{\mu}{m} \omega+\xi^{2} \sin \theta \cos \theta-\frac{g}{R} \sin \theta
$$

is IUBIBSS on $C$ with $\theta$ seen as an input. We will conclude IUBIBSS by proving the stronger property of IISS. Consider the function:

$$
U=\frac{1}{2}\left(\omega_{1}-\omega_{2}\right)^{2}
$$

Its time derivative is given by:

$$
\begin{aligned}
\dot{U}= & \left(\omega_{1}-\omega_{2}\right)\left[-\frac{\mu}{m}\left(\omega_{1}-\omega_{2}\right)+\xi^{2} \sin \theta_{1} \cos \theta_{1}\right. \\
& \left.-\frac{g}{R} \sin \theta_{1}-\xi^{2} \sin \theta_{2} \cos \theta_{2}+\frac{g}{R} \sin \theta_{2}\right] \\
\leq & -\frac{\mu}{m}\left(\omega_{1}-\omega_{2}\right)^{2}+\left|\omega_{1}-\omega_{2}\right| \mid \xi^{2} \sin \theta_{1} \cos \theta_{1} \\
& -\frac{g}{R} \sin \theta_{1}-\xi^{2} \sin \theta_{2} \cos \theta_{2}+\frac{g}{R} \sin \theta_{2} \mid \\
\leq & -\frac{\mu}{m}\left(\omega_{1}-\omega_{2}\right)^{2}+\left|\omega_{1}-\omega_{2}\right| L\left|\theta_{1}-\theta_{2}\right| \\
= & -\frac{\mu}{2 m}\left(\omega_{1}-\omega_{2}\right)^{2} \\
& +\left(-\frac{\mu}{2 m}\left(\omega_{1}-\omega_{2}\right)^{2}+\left|\omega_{1}-\omega_{2}\right| L\left|\theta_{1}-\theta_{2}\right|\right)
\end{aligned}
$$

where the second inequality follows from the fact that $\xi^{2} \sin \theta \cos \theta-\frac{g}{R} \sin \theta$ is a smooth function defined on the convex compact set $\pi_{\theta}(C)$ and is thus globally Lipschitz on $\pi_{\theta}(C)$ (since its derivative is continuous and thus bounded on $\pi_{\theta}(C)$ ) with Lipschitz constant $L$. We now note that the condition:

$$
\left|\omega_{1}-\omega_{2}\right|>\frac{2 m L}{\mu}\left|\theta_{1}-\theta_{2}\right|
$$

makes the second term in (8) negative from which we conclude the following implication:

$\left|\omega_{1}-\omega_{2}\right|>\frac{2 m L}{\mu}\left|\theta_{1}-\theta_{2}\right| \quad \Longrightarrow \quad \dot{U} \leq-\frac{\mu}{2 m}\left(\omega_{1}-\omega_{2}\right)^{2}$ 


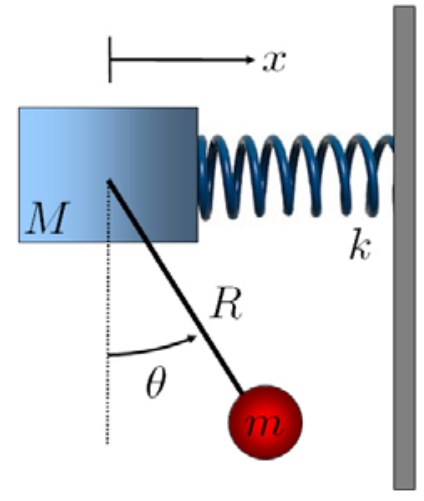

Fig. 2. A graphical representation of the pendulum on a cart mounted to a spring.

showing that $U$ is an IISS Lyapunov function. We can thus reduce (7) to:

$$
\dot{\omega}=-\frac{\mu}{m} \omega .
$$

Projected trajectories of the full-order system as compared with trajectories of the reduced system can be seen in Figure 1 ; here $\mu=m=1$ and $\xi=0.1$. Note that as $R \rightarrow \infty$, the reduced system converges to the full-order system (or the full-order system effectively becomes decoupled).

Example 2: We now consider a pendulum attached to a cart with is mounted to a spring (see Figure 2). For this example, there are the following parameters:

$$
\begin{aligned}
M & =\text { mass of the cart } \\
m & =\text { mass of the pendulum, } \\
R & =\text { length of the rod, } \\
k & =\text { spring stiffness, } \\
g & =\text { acceleration due to gravity } \\
d & =\text { friction constant for the cart } \\
b & =\text { friction constant for the pendulum. }
\end{aligned}
$$

The equations of motion are given by:

$$
\begin{aligned}
\dot{x}= & v \\
\dot{\theta}= & \omega \\
\dot{v}= & \frac{1}{M+m \sin ^{2} \theta}\left(m R \omega^{2} \sin \theta+m g \sin \theta \cos \theta\right. \\
& \left.-k x-d v+\frac{b}{R} \cos \theta\right) \\
\dot{\omega}= & \frac{1}{R\left(M+m \sin ^{2} \theta\right)}\left(-m R \omega^{2} \sin \theta \cos \theta\right. \\
& -(m+M) g \sin \theta+k x \cos \theta+d v \cos \theta \\
& \left.-\left(1+\frac{M}{m}\right) \frac{b}{R} \omega\right)
\end{aligned}
$$

where $x$ is the position of the cart, $v$ its velocity, $\theta$ is the angular position of the pendulum and $\omega$ its angular velocity.

If $\pi_{(x, v)}: \mathbb{R}^{4} \rightarrow \mathbb{R}^{2}$ is the projection $\pi_{(x, v)}(x, \theta, v, \omega)=$ $(x, v)$ and $X$ is the vector field as defined in (9), the goal is to reduce $X$ to $\mathbb{R}^{2}$ by eliminating the $\theta$ and $\omega$ variables and thus obtaining $Y$ defined by:

$$
\begin{aligned}
\left(\begin{array}{c}
\dot{x} \\
\dot{v}
\end{array}\right) & =Y(x, v) \\
& =T_{(x, \theta, v, \omega)} \pi_{(x, v)} \cdot X(x, 0, v, 0) \\
& =\left(\begin{array}{c}
v \\
-\frac{1}{M}(d v+k x)
\end{array}\right) .
\end{aligned}
$$

The objective is now to show that $X$ and $Y$ are approximately $\pi_{(x, v)}$-related. In particular, note that the reduced system, $Y$, is linear while the full-order system, $X$, is very nonlinear. This will be discussed in more detail after proving that they are in fact approximately related.

Stability of $X$, and in particular fiberwise stability, can be proven as in the previous example by noting that $X$ is Hamiltonian for $d=b=0$ and using the Hamiltonian as a Lyapunov function $V$. Consider now the control system:

$$
\begin{aligned}
F((x, v),(\theta, \omega))= & T \pi_{(x, v)} \cdot X(x, \theta, v, \omega) \\
= & \frac{1}{M+m \sin ^{2} \theta}\left(m R \omega^{2} \sin \theta-k x\right. \\
& \left.+m g \sin \theta \cos \theta-d v+\frac{b}{R} \cos \theta\right)
\end{aligned}
$$

with $\theta$ and $\omega$ regarded as inputs. To show that $F$ is IUBIBSS we first rewrite (10) in the form:

$$
\begin{aligned}
& F((x, v),(\theta, \omega))= \\
& \quad \frac{1}{M+m}\left(m R \omega^{2} \sin \theta-k x-d v-m R \dot{\omega} \cos \theta\right)
\end{aligned}
$$

and consider the following IISS candidate Lyapunov function:

$$
U=\frac{1}{2(m+M)}\left(x_{1}-x_{2}\right)^{2}+\frac{1}{2}\left(v_{1}-v_{2}\right)^{2} .
$$

Its time derivative is given by:

$$
\begin{aligned}
\dot{U}= & -\frac{d}{m+M}\left(v_{1}-v_{2}\right)^{2}+\frac{m R}{m+M}\left(\omega_{1}^{2} \sin \theta_{1}\right. \\
& \left.-\dot{\omega}_{1} \cos \theta_{1}-\omega_{2}^{2} \sin \theta_{2}+\dot{\omega}_{2} \cos \theta_{2}\right)\left(v_{1}-v_{2}\right) .
\end{aligned}
$$

Using an argument similar to the one used for the previous example, we conclude that:

$$
\left|v_{1}-v_{2}\right| \geq \frac{2 m R L}{d}\left|\left(\theta_{1}, \omega_{1}, \dot{\omega}_{1}\right)-\left(\theta_{2}, \omega_{2}, \dot{\omega}_{2}\right)\right|,
$$

with $L$ the Lipschitz constant of the function $\omega^{2} \sin \theta-$ $\dot{\omega} \cos \theta$, implying:

$$
\dot{U} \leq-\frac{d}{2(m+M)}\left(v_{1}-v_{2}\right)^{2},
$$

thus showing that $X$ is IISS and in particular also IUBIBSS. That is, we have established that $X$ and $Y$ are approximately $\pi_{(x, v)}$-related.

In order to illustrate some of the interesting implications of approximate reduction, we will compare the reduced system, $Y$, and the full-order system, $X$, in the case when $R=m=$ 

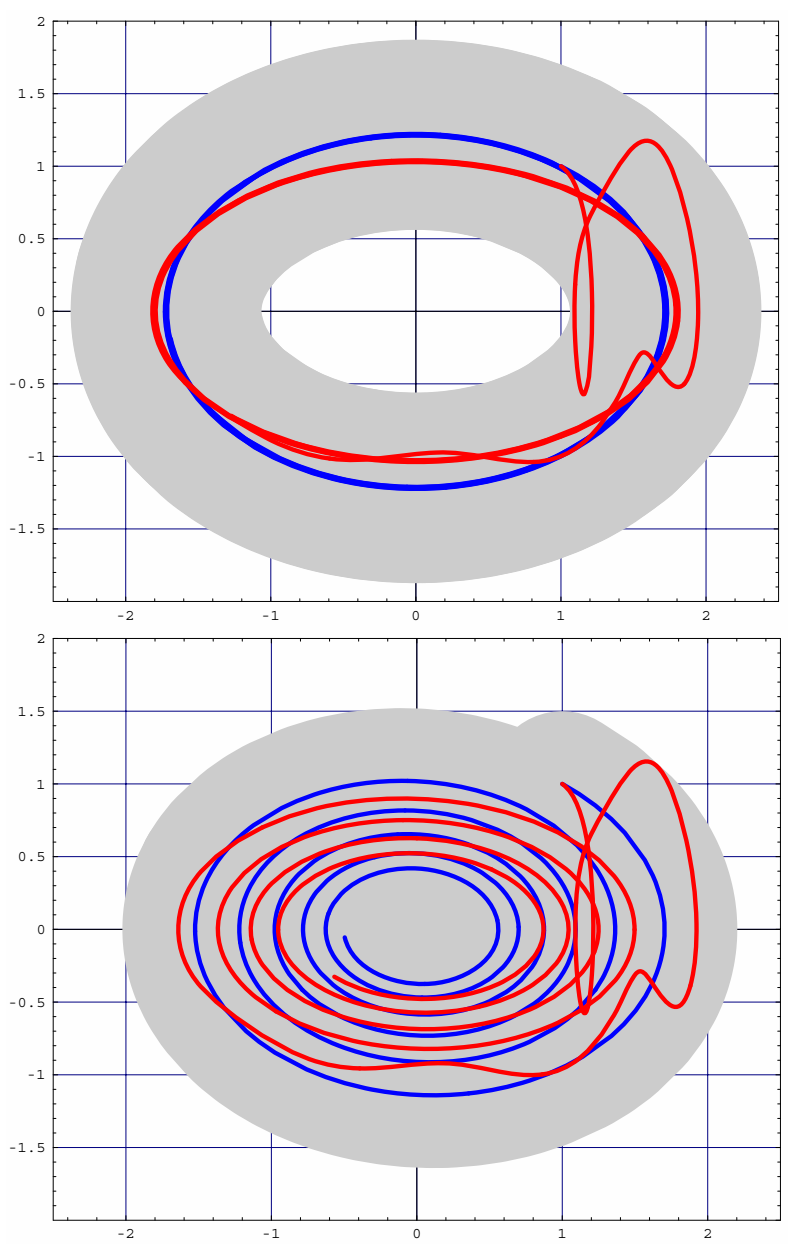
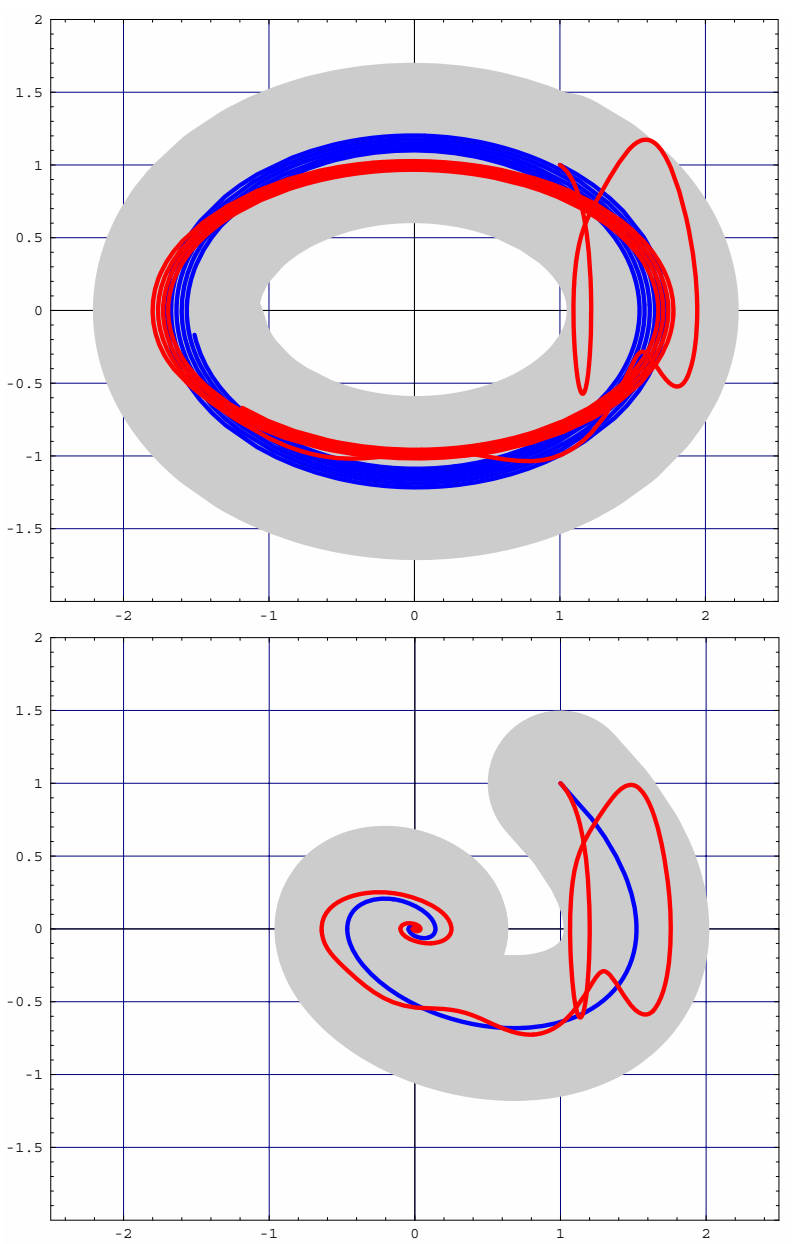

Fig. 3. A projected trajectory of the full-order system (red) and a trajectory for the reduced system (blue) for $d=0.001,0.01,0.1,1$ (from left to right and top to bottom, respectively).

$k=b=1$ and $M=2$. It follows that the equations of motion for the reduced system are given by the linear system:

$$
\left(\begin{array}{c}
\dot{x} \\
\dot{v}
\end{array}\right)=\left(\begin{array}{cc}
0 & 1 \\
-\frac{1}{2} & d
\end{array}\right)\left(\begin{array}{l}
x \\
v
\end{array}\right),
$$

so we can completely characterize the dynamics of the reduced system: every solution spirals into the origin. This is in stark contrast to the dynamics of $X$ (see (9)) which are very complex. The fact that $X$ and $Y$ are approximately related, and more specifically Theorem 1, allows us to understand the dynamics of $X$ through the simple dynamics of $Y$. To be more specific, because the distance between the projected trajectories of $X$ and the trajectories of $Y$ is bounded, we know that the projected trajectories of $X$ will "essentially" be spirals. Moreover, the friction constant $d$ will directly affect the rate of convergence of these spirals. Examples of this can be seen in Figure 3 where $d$ is varied to affect the convergence of the reduced system, and hence the full order system.

\section{REFERENCES}

[AMR88] R. Abraham, J. Marsden, and T. Ratiu. Manifolds, Tensor Analysis and Applications. Applied Mathematical Sciences. Springer-Verlag, 1988.
[Ang02] D. Angeli. A lyapunov approach to incremental stability properties. IEEE Transactions on Automatic Control, 47(3):410421, 2002.

[ASG00] A. C. Antoulas, D. C. Sorensen, and S. Gugercin. A survey of model reduction methods for large-scale systems. Contemporary Mathematics, 280:193-219, 2000.

[BDG96] C. L. Beck, J. Doyle, and K. Glover. Model reduction of multidimensional and uncertain systems. IEEE Transactions on Automatic Control, 41(10):1466-1477, 1996.

[BKMM96] A.M. Bloch, P.S. Krishnaprasad, J.E. Marsden, and R. Murray. Nonholonomic mechanical systems with symmetry. Archive for Rational Mechanichs and Analysis, 136(1):21-99, 1996.

[BM00] A. Bacciotti and L. Mazzi. A necessary and sufficient condition for bounded-input bounded-state stability of nonlinear systems. SIAM Journal on Control and Optimization, 39(2):478-491, 2000.

[MR99] Jerrold E. Marsden and Tudor S. Ratiu. Introduction to Mechanics and Symmetry. Number 17 in Texts in Applied Mathematics. Springer-Verlag, 2nd edition, 1999.

[MSVS85] Giuseppe Marmo, Alberto Simoni, Bruno Vitale, and Eugene J. Saletan. Dynamical Systems. John Wiley \& Sons, 1985.

[MW74] J. E. Marsden and A. Weinstein. Reduction of symplectic manifolds with symmetry. Reports on Mathematical Physics, 5:121-120, 1974.

[vdS81] A. van der Schaft. Symmetries and conservation laws for Hamiltonian systems with inputs and outputs: A generalization of Noether's theorem. Systems and Control Letters, 1:108-115, 1981. 